\title{
SUPPLEMENT
}

\section{Prevention of anterior cruciate ligament injury in the female athlete}

\author{
Holly Jacinda Silvers, Bert R Mandelbaum
}

\author{
See end of article for \\ authors' affiliations \\ ..................... \\ Correspondence to: \\ Ms H J Silvers, Santa Monica \\ Orthopaedic Sports \\ Medicine/Research \\ Foundation, Suite 350, \\ 1919 Santa Monica \\ Boulevard, Santa Monica, \\ CA 90404, USA; \\ hollysilverspt@aol.com
}

Accepted 13 March 2007

Published Online First

3 July 2007
Br J Sports Med 2007;41(Suppl I):i52-i59. doi: 10.1136/bjsm.2007.037200
$\mathrm{T}$ he anterior cruciate ligament (ACL) is one of the major stabilising intracapsular ligaments in the knee joint. It prevents excessive anterior translation of the tibia on the femur. In addition, it plays a secondary role of limiting internal rotation of the tibia. It is housed in the intercondylar notch of the femur. The proximal attachment is the posterior medial surface of the lateral femoral condyle, with distal attachment to the anterior portion of the intercondylar eminence of the tibia. There are two distinct bundles in the ACL joint: (1) the anteromedial portion, which is taut in knee flexion; (2) the posterolateral portion, which is taut in knee extension.

The ACL works collectively with the posterior cruciate ligament to stabilise the knee during dynamic movement. The latter is attached to the posterior portion of the intercondylar eminence of the tibia and passes forward to attach to the medial condyle of the femur. The medial collateral ligament is attached to the medial femoral condyle and the medial surface of the tibia. The lateral collateral ligament is attached to the lateral femoral condyle and the lateral portion of the head of the fibula. These two ligaments are extracapsular and provide stability to the knee joint in the frontal plane during varus and valgus loads.

\section{HOW IS THE ACL RUPTURED?}

Injuries to the knee joint are common. Ligamentous or meniscal structures are typically damaged during knee injury. Ligaments are usually torn when an excessive external force is applied to the limb, whereas meniscal injuries usually occur as the result of forces generated within the limb (torsional). However, unlike most ligamentous structures in the body, the ACL can be injured without external force being applied to the knee joint. In the USA alone, about 250000 ACL injuries occur annually. This translates into a 1 in 3000 chance that a member of the general population will sustain an ACL injury. The fiscal burden of such an injury is quite large; when the costs of MRI, reconstructive surgery, postoperative bracing and rehabilitation are considered, the average annual cost exceeds 2 billion dollars. In addition, the psychological impact of such an injury can be quite devastating. Typically, an athlete will miss 6-9 months of competitive play as a result of the injury itself and reconstructive surgery and rehabilitation. However, this estimate does not reflect the large dichotomy that exists when the epidemiological data are stratified with respect to gender. Since Title IX of the Education Amendments of 1972 was passed, allowing equal opportunity for female participation in sport, there has been an exponential surge of female participation in organised sports. With this increase in athletic participation, the number of injuries sustained by women has increased. Female athletes have a 2-10-fold higher incidence of ACL injuries than their male counterparts. Arendt and Dick ${ }^{1}$ examined the increased incidence of ACL injury among NCAA division I athletes participating in basketball and soccer over a 5 -year period. The injury rate was recorded and analysed per athlete-exposure, where one practice session or game counted as an exposure. The average ACL injury rate was 0.31 per 1000 athlete-exposures for female soccer and 0.29 per 1000 athlete exposures for female basketball. This compares with 0.13 per 1000 athlete-exposures for male soccer and 0.07 per 1000 athlete-exposures for male basketball. These epidemiological data on ACL injury rates show the discrepancy between the genders.

Complete ACL injuries can lead to chronic knee pathology, including instability, secondary injury to the meniscii and articular cartilage, and early onset of osteoarthritis. About $66 \%$ of all patients with a complete ACL injury have damage to the menisci and the articular cartilage of the femur, patella and/or tibia. This injury, coupled with the risk of secondary injury, can significantly decrease the ability of a patient to achieve activities of daily living and affect their quality of life. The decision to delay surgical reconstruction of a ruptured ACL can significantly increase the risk of secondary injury. Seitz et al noted that $65 \%$ of ACL-deficient patients sustained a secondary meniscal injury within 2.5 years of the initial injury.

Despite the most earnest efforts of orthopaedic surgeons to preserve the integrity of the knee joint during ACL reconstructive surgery, patients who have had this surgery continue to present with degenerative changes of the articular cartilage and the inevitable early onset of osteoarthritis. Lohmander et $a l^{3}$ completed a 12-year longitudinal study following-up female athletes who had ACL reconstruction after sustaining an injury 
while playing soccer. They found that 55 (82\%) had radiographic changes in their index knee, and 34 (51\%) fulfilled the criterion for radiographic osteoarthritis of the knee. The mean age of the subjects in the study was 31 . The implications of this research are ominous, hence the increased need for prevention of these injuries in the first place.

\section{MECHANISM OF INJURY}

The mechanism of ACL injury has been elucidated. There are two types: contact and non-contact. Over two thirds (70\%) of all reported ACL injuries are non-contact in origin, the remainder involving contact from an outside force such as an opposing player, a goalpost or another object on the field/court.

The mechanism of non-contact ACL injury in field/court sports commonly involves a one-step/stop deceleration, cutting movements, sudden change of direction, landing from a jump with inadequate knee and hip flexion (at or near full extension), or a lapse of concentration (resulting from an unanticipated change in the direction of play). Non-contact ACL injuries typically occur during a deceleration manoeuvre combined with a change of direction while the foot is in a closed-chain position. While the foot is in a closed-chain position and pronated, the tibia is internally rotated, and the knee is at or near full extension (0-20 of flexion); if the athlete attempts to change direction, the result is an excessive torsional force that can potentially strain or rupture the ACL.

In downhill alpine skiing, the mechanism of injury is slightly different. Three common causes of ACL injury have been discussed in the literature. The first is the "phantom-foot mechanism" where a skier falls backwards with the knee flexed past $90^{\circ}$, and the tibia rotates internally. The combination of a strong quadriceps contraction applying an excessive anterior force to the proximal tibia with a rigid ski boot (distally) that fails to release may lead to an ACL sprain or tear. The second mechanism involves the skier landing on the tail of the ski. The stiffness of the posterior shell of the boot combined with a strong quadriceps contraction applying an excessive anterior migration of the tibia may lead to an ACL injury. The third mechanism typically involves male downhill skiers skiing under poor conditions and/or at high velocity. This mechanism occurs when the anteromedial edge of the ski becomes impacted under snow. The involved limb begins to abduct and externally rotate while the skiers' momentum carries him/her forward.

\section{PREVENTION}

A consensus group comprising doctors, surgeons, physiotherapists and certified athletic trainers met in Hunt Valley, Maryland in 1999 to discuss the "epidemic" of ACL injuries in the female athlete. Four categorical risk factors were identified during the attempt to determine the aetiology of the increased rate of ACL injuries in the female athlete. The risk factors were examined independently in order to determine the primary factors involved:

- Anatomy

- Hormones

- Environment

- Biomechanical and neuromuscular factors

The same group of researchers met again in Atlanta, Georgia in January 2005 to re-evaluate the identified risk factors and to determine what progress had been made since the inaugural meeting in 1999.

\section{Anatomy}

Multiple differences in anatomical risk factors exist between the genders. The typical female athlete has increased femoral anteversion, an increased Q angle, excessive tibial torsion, and excessive subtalar pronation compared with her male counterpart. In addition, the size of the intercondylar notch in the femur and the actual diameter of the ACL itself are smaller. Impingement of the ACL against the lateral portion of the medial intercondylar notch has been proposed as a potential anatomical cause of ACL injury. Typically, men have a wider Ushaped intercondylar notch, whereas women have a narrower cresting wave (A-shape) notch. It has been suggested that, owing to the narrower geometry of the female intercondylar notch, the ACL may impinge on the medial border of the lateral femoral condyle when combined with a valgus load. However, the cross-sectional area of the female ACL is significantly smaller than in men. Perhaps, in the female athlete, having a smaller ACL in a narrow intercondylar notch provides some protection to the ACL. No studies to date have indicated that there is a direct correlation between ACL size and ACL injury rates. Shelbourne and Lootwyk ${ }^{4}$ studied patients who had received autogenous patella tendon graft ACL reconstructions and divided them into two groups: narrow notches and wide notches. Intercondylar notches $>16 \mathrm{~mm}$ were defined as wide, and those $<15 \mathrm{~mm}$ were defined as narrow. The notch width in female patients was narrower than in male counterparts of the same height. However, the results did not indicate any correlation between gender and the rate of injury to the contralateral ACL or the re-rupture rate. Anatomical risk factors may play a small role in the incidence of non-contact ACL injury; however, they may have a more direct impact when the body is dynamically moving. Studies assessing incidence in relation to anatomical risk factors and multiplanar dynamic movement patterns are necessary.

\section{Environment}

Environmental risk factors are extrinsic factors that include prophylactic and functional knee bracing, footwear choice, playing surface and weather (climate).

\section{Prophylactic and functional knee bracing}

No studies have conclusively demonstrated the effectiveness of functional knee braces in preventing non-contact ACL injuries. A study by Deppen ${ }^{5}$ examined the use of prophylactic knee bracing in high school football players. The injury rates of eight high school football teams were analysed comparing those who wore prophylactic knee braces with those who did not. Over a 4-year period, 23 knee injuries occurred in 21640 exposures in the braced group compared with 26 knee injuries in 19484 exposures in the non-braced group. There was no statistically significant difference in the rate of knee injury or the severity of injuries between the two groups. Because of the financial implications of prophylactic bracing and the apparent similarities between the rates of injury in the braced and non-braced group, it is recommended that the medical community does not advocate the use of prophylactic bracing in this population.

\section{Footwear}

In 1974, Torg et $a l^{6}$ developed a quantitative measurement entitled "release coefficient" to describe the force/weight ratio of shoe-surface interaction. This work was reinforced by Heidt et al, ${ }^{7}$ who found that $73 \%$ of the 15 different types of athletic shoes tested had an "unsafe" or "probably unsafe" rating. When considering shoe design, it is important to remember that, although increased friction coefficient may enhance performance, it may also inadvertently increase ligamentous injury. Ekstrand and $\mathrm{Nigg}^{8}$ noted that there is an optimal range to be incorporated into shoe design: one that will minimise rotational friction to avoid injury yet optimise transitional 
friction to allow peak performance in activities such as cutting and decelerating.

\section{Playing surface}

Playing surface and shoes must be considered when determining whether or not these factors can independently increase the rate of non-contact ACL injuries in women. A recent study of the incidence of ACL injury in European team handball found that injury rates on wooden floors (parquet), which generally have lower friction, are lower than those on artificial floors, which generally have higher friction. A total of 174 ACL injuries were recorded over 11 seasons. The floor types (wood or artificial) for all games were recorded. Nine injuries occurred in men (mean (SD) incidence $0.24(0.09)$ ) and 44 in women (mean (SD) incidence $0.77(0.04)$ ). In the men, four injuries occurred on wooden floors $(0.32(0.13))$ and five on artificial floors $(0.20(0.12))$. In the women, eight injuries occurred on wooden floors $(0.41(0.09))$ and 36 on artificial floors $(0.96$ (0.04)). The results indicate that the risk of ACL injury for women is higher than for men on artificial floors than on wooden floors. Uneven playing surfaces may play a role in ACL incidence. In the study of Huston et al, ${ }^{9}$ patients reported landing or stepping on an uneven surface (inconsistency in grass or another player) at the time of injury. In addition, irrigation of the field may affect the rate of ACL injury. Scranton et al $^{10}$ identified 61 non-contact ACL injuries in 22 National Football League (NFL) teams over the course of four seasons. The variables of surface, shoe type, playing conditions and shoe spatting were identified for each ACL injury. Forty non-contact injuries occurred in conventional cleated shoes on natural grass, and 21 occurred on an artificial surface. Injury rates on game day exceeded rates in practice: $47.5 \%$ occurred during game-day exposures despite the fact that the practice versus game-day exposure was 5:1. Of these injuries, $95.2 \%$ occurred on a dry field.

\section{Weather (climate)}

Orchard and Powell ${ }^{11}$ analysed 5910 NFL team games to determine if a correlation existed between knee and ankle sprains, playing surface, and the weather conditions on the day of the game. He found a lower risk of significant ankle sprains for games in natural grass stadiums compared with indoor domes (with AstroTurf). In addition, there was also a lower incidence of significant knee sprains on grass than in domes which was directly related to cold and wet weather on grass. In open (outdoor) AstroTurf stadiums, cold weather was associated with a lower risk of ACL injuries compared with hot weather in the same stadiums. ACL incidence was lower during the later (cooler) months of the season in open stadiums (both AstroTurf and natural grass) but not in domes. The researchers concluded that cold weather is associated with lower knee and ankle injury risk in outdoor stadiums (both natural grass and AstroTurf). This reduction is directly related to lower shoesurface friction coefficients.

\section{Hormones}

There has been increasing speculation that monthly hormonal changes may increase a female athlete's susceptibility to ligamentous injury. The menstrual cycle can be subdivided into three distinct phases, based on a mean cycle of 28 days. In the follicular phase (days 1-9), low concentrations of both progesterone and oestrogen are present until late follicular phase, when a spike in oestrogen is seen. The ovulatory phase (days 10-14) is preceded by a continuation of the spike of oestrogen. In the luteal phase (days 15-28), there is a rise in progesterone, and a rise in relaxin in the second half of this phase. Fluctuations in progesterone, oestrogen and relaxin throughout the menstrual cycle have been studied to determine their effect on the integrity of the ACL. Oestrogen, progesterone and relaxin receptor sites have been found in the ACL.

The increases in oestrogen and relaxin have been shown to coincide with a subsequent $40 \%$ decrease in the rate of collagen synthesis. Yu et al $^{12}$ simulated the effects of $17 \beta$-oestradiol on the rate of cell proliferation and precollagen concentrations in female ACL fibroblasts throughout the menstrual cycle in an in vitro study. Samples of ACL from a healthy female subject were treated with $17 \beta$-oestradiol on the following days of the menstrual cycle: 1, 3, 7, 10 and 14. Cell proliferation and precollagen concentrations were used to determine the rate of collagen synthesis. An inverse correlation between the ACL fibroblasts and the concentration of $17 \beta$-oestradiol was found. As the $17 \beta$-oestradiol dose increased, the number of fibroblasts in the ACL decreased in a dose-dependent fashion. The researchers proposed that $17 \beta$-oestradiol released during the normal menstrual cycle may affect the incidence of ACL injury in female athletes through its direct effect on collagen synthesis.

Slauterbeck et $a l^{13}$ used a rabbit model to determine the effects of oestrogen on the load to failure of the ACL. They showed a decrease in tensile properties of the ACL and a subsequent decrease in the ACL failure load in the oestrogen treatment group (rabbits) compared with the controls.

With regard to ACL injury in relation to phase of the cycle, Wojtys et $a l^{14}$ noted a statistically significant increase in ACL injury while athletes were in the ovulatory phase (days 10-14) of the menstrual cycle. Myklebust et $a{ }^{15}{ }^{15}$ in contrast with the findings of Wojyts et al, found that the ACL injury rate decreased between days 8 and 14. However, there is some discrepancy between collection methods for the hormonal assays (saliva, urine or blood). Furthermore, obtaining an account of menstrual status from an athlete retrospectively may lack accuracy. Therefore, there is no conclusive evidence directly linking an increase in ACL injury to a predictable time in the menstrual cycle. Further studies investigating phase of the menstrual cycle, larger sample sizes, and independent hormonal assays at various times throughout the menstrual cycle should be encouraged.

\section{Biomechanical and neuromuscular factors}

With regard to environmental, anatomical and hormonal risk factors, there is no conclusive evidence that any one single risk factor correlates directly with an increase in ACL injury in female athletes. Therefore, the emphasis has turned to biomechanical risk factors and the use of neuromuscular and proprioceptive intervention programmes to address potential biomechanical deficits.

Neuromuscular control of the knee involves a complex interaction between the afferent and efferent neurological system and the muscles that control the knee joint. The feedforward mechanism is a system used to anticipate external forces or loads in order to stabilise the joint, thus protecting the inherent structures. Proprioception is described as the acquisition of stimuli by peripheral receptors in addition to the conversion of mechanical stimuli into a neural signal that is transmitted along afferent pathways of the sensorimotor system. Proprioception does not include central nervous system processing of the incoming afferent signal, nor does it include control of efferent (outgoing) motor signals. However, this "proprioceptive" information is crucial for optimal motor performance. It is delivered to every motor control centre and is used to garner information about joint position and kinaesthesia (joint motion) in space in order to elicit active and reflexive movement. Neuromuscular control is defined as the unconscious efferent response to an afferent signal about 
dynamic joint stability. The afferent proprioceptive signals that elicit motor control can be distinguished by their role: feedback or feedforward. Feedback mechanisms are a result of afferent input (force to the joint) and are reflexive in nature. The time to elicit such a reaction is longer, thus it is thought to be more heavily involved with maintaining posture and slow movement. Feedforward mechanisms are a result of preactivated preparatory activation of muscle. Several research studies have indicated that proprioceptive activities may have a major role in injury reduction.

Muscular strength and recruitment patterns are crucial to knee stability. Quadriceps to hamstring strength ratios have been examined thoroughly in the literature. The quadriceps muscle serves as an antagonist to the ACL: because of its attachment site, it increases the anterior shear force on the tibia. The hamstring muscle acts as an agonist to the ACL, as it reinforces the ligament by preventing the excessive anterior translation of the tibia. If the hamstring shows weakness or a delay in contraction time in comparison with the quadriceps, the ACL may be at increased risk of injury, leading to tensile failure.

Landing from a jump with minimal hip and knee flexion increases the load transmitted to the knee and increases the shear force from the quadriceps, thus stressing the ACL. More et $a l^{16}$ studied a cadaveric model that incorporated quadriceps and hamstring muscle loads to simulate the squat exercise. When the hamstring was loaded, anterior tibial translation during flexion was significantly reduced in addition to a reduction in internal tibial rotation during flexion. Hamstring muscle activity during a squat functions synergistically with the ACL to provide anterior knee stability. McLean et al ${ }^{17}$ compared knee kinematics and gender in 30 high-performance athletes performing side-cutting manoeuvres. Women displayed increased inter-trial variability in axial internal rotation patterns during cutting compared with men. However, gender was not the main determining factor. Instead, the differences in axial rotation were directly related to level of experience. It is important to note that these subjects were high-performance athletes, which may have resulted in selection bias. In a followup study, McLean et $a l^{18}$ studied 10 male and 10 female athletes performing cutting manoeuvres with random perturbations at initial contact $(n=5000)$. Injury to the ACL in the sagittal plane was defined as incurring an anterior drawer force greater than $2000 \mathrm{~N}$. The researchers found that neuromuscular perturbations produced significant increases in external knee anterior force, valgus moments and internal rotation moments. During the study, the anterior drawer force never exceeded $2000 \mathrm{~N}$ in any model. Valgus loads reached values that were high enough to rupture the ligament, and occurred more often in women than in men. McLean et al concluded that sagittal plane knee joint forces cannot rupture the anterior cruciate ligament during sidestep cutting, primarily because the muscle and joint mechanics and external ground reaction forces in this plane protect the upward limit of ligament loads. They suggested that valgus loading is a more likely injury mechanism, especially in women.

In contrast, Malinzak et al ${ }^{19}$ compared knee motion patterns in recreational male and female athletes. Three-dimensional coordinates and electromyographic data were collected for knee flexion-extension, valgus-varus and internal-external rotation angles. Female athletes showed less knee flexion and greater knee valgus when landing from a jump and with cutting manoeuvres. The study also found that female athletes showed greater quadriceps activity (electromyographic analysis) in concert with decreased hamstring activity. In addition, the frequency and intensity of hamstring activity was less in female than male athletes. Female athletes typically contracted their hamstring fibres $50 \mathrm{~ms}$ more slowly than their male counterparts (200 vs $150 \mathrm{~ms}$ ) and with less intensity (55.2\% vs $71.8 \%$ at initial contact).

\section{Prevention}

ACL injury prevention programmes focusing on skiing, basketball, European team handball and soccer have been performed in the past with overall reduction in severe ACL injuries ranging from $60 \%$ to $89 \%$.

Henning ${ }^{20}$ implemented a prevention study in two division I basketball programmes over a course of 8 years geared at changing player technique: stressing knee flexion upon landing, using accelerated rounded turns, and deceleration with a multi-step stop. He noted an $89 \%$ reduction in the incidence of ACL injuries in his intervention group.

Caraffa et $a^{21}$ implemented a proprioceptive balance training programme using 600 semiprofessional and amateur soccer players in Italy. The study consisted of a 20-min training programme divided into five phases of increasing difficulty. The prospective study was completed over the duration of three complete soccer seasons. An incidence of 1.15 ACL injuries per team per year was found in the control group compared with 0.15 injuries per team per year in the trained athletes. These results show an overall $87 \%$ decrease in ACL injuries compared with the control group.

Hewett et al ${ }^{22}$ completed a prospective analysis of 1263 male and female athletes from various sports using a neuromuscular training programme. They used a 6-week intervention programme consisting of stretching, plyometrics and weight training with emphasis on proper alignment and technique. The incidence of serious knee injury was 2.4-3.6 times higher in the untrained group than in the trained group. When the rate of non-contact ACL injuries was examined, five untrained female athletes sustained ACL injuries (relative injury incidence 0.26), no trained females sustained an ACL injury (0), and one male athlete sustained an ACL injury (0.05).

Ettlinger et al ${ }^{23}$ implemented the "guided discovery" technique in Vermont which focused on avoiding high-risk behaviour and positioning (ie, "phantom foot"), recognising potentially dangerous skiing situations, and responding quickly to unfavourable conditions. During the 1993-1994 ski season, 4700 ski instructors and patrollers completed the comprehensive training programme at $20 \mathrm{ski}$ areas throughout the USA. As a result, the rate of serious knee injuries decreased by $62 \%$ among the trained personnel compared with those who did not participate in the training programme.

Heidt et al $^{24}$ studied 300 female adolescent soccer players aged 14-18 years of age over a 1-year period. Forty two athletes participated in the Frappier acceleration training program, a 7week preseason training programme consisting of strength training, flexibility, sports-specific cardiovascular exercise, plyometrics, and sports cord drills. They found that the trained group had fewer ACL injuries (2.4\%) than the age-matched control group $(3.1 \%)$.

Myklebust et $a l^{15}$ instituted a proprioceptive training programme for an elite female team of handball players. This fivephase training programme consisted of floor exercises, wobble board activities and a balance mat, and was performed 23 times a week over the course of 5-7 weeks preseason and once a week in season. Fifty eight teams (855 players) participated in the first season (1999-2000) and 52 teams (850 players) in the second season (2000-2001). Sixty teams (942 players) in the 1998-1999 season served as the control There were 29 ACL injuries in the control season, 23 ACL injuries in the first intervention season, and 17 injuries in the second intervention season. 


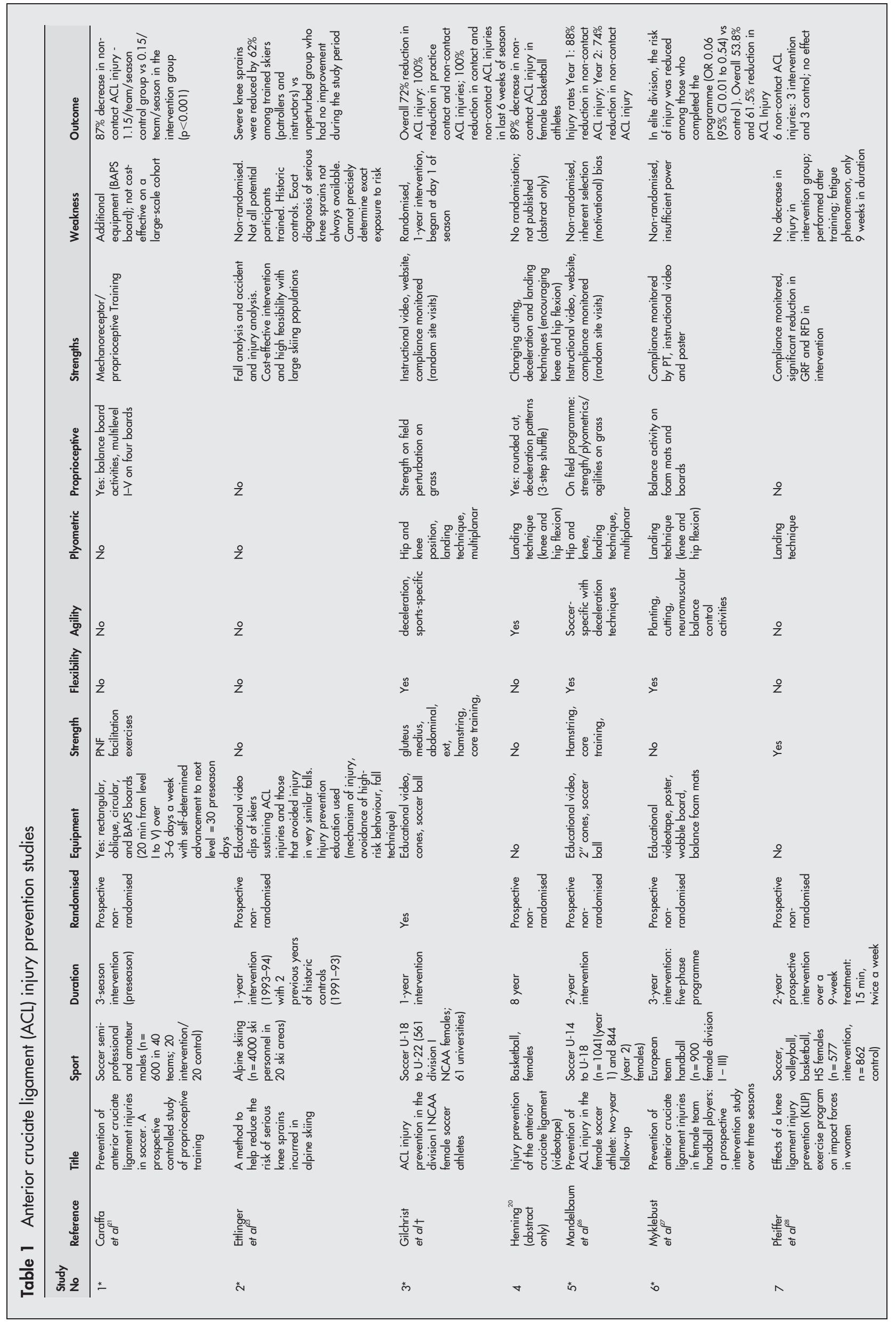




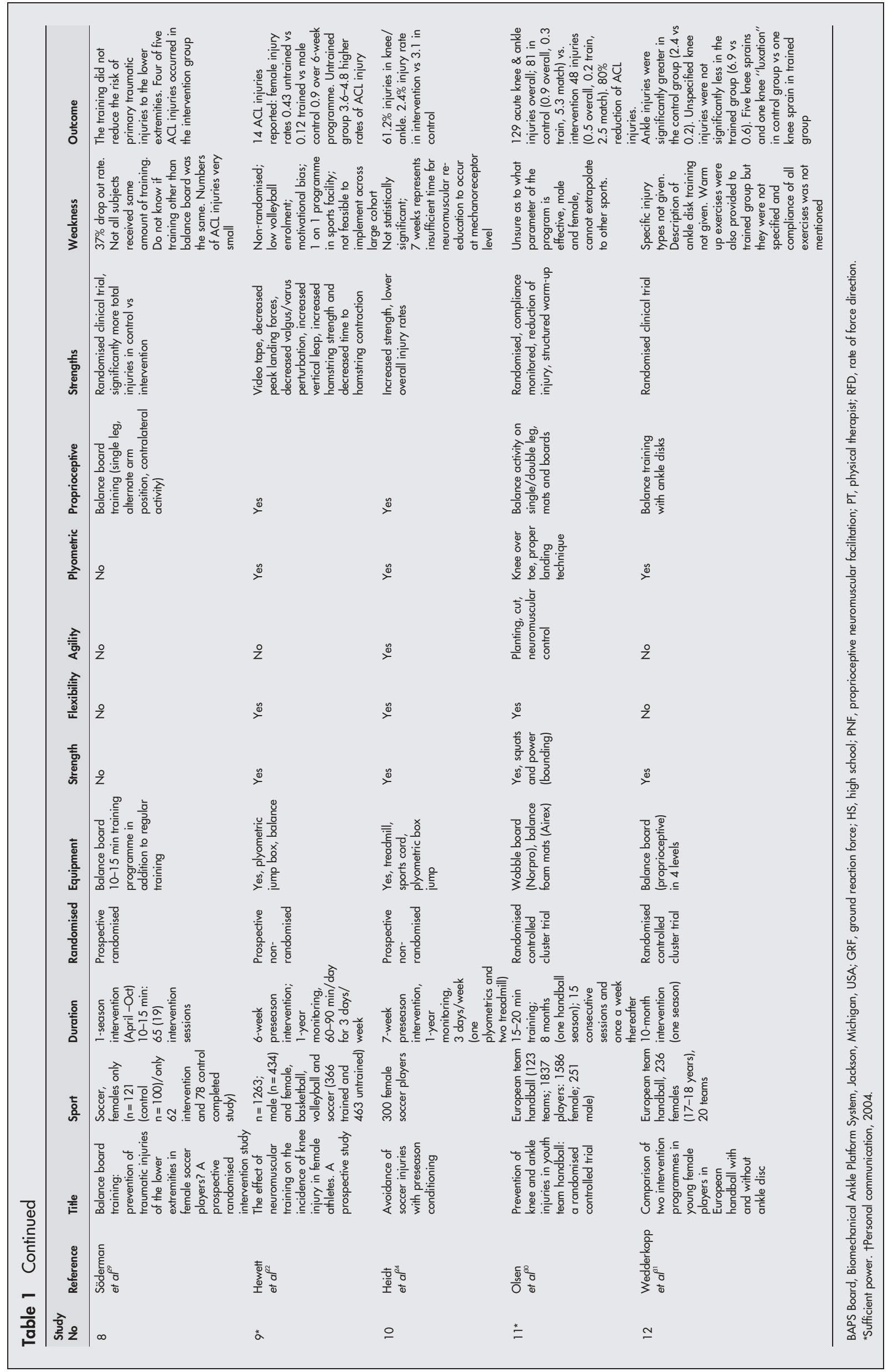


Table 2 Relation of intervention strategies to specific risk factors for injury of the anterior cruciate ligament

\begin{tabular}{lll}
\hline Position & Intervention strategy & Method \\
\hline Extended knee at initial contact & Knee flexion & Concentric hamstring control and soft landing \\
Extended hip at initial contact & Hip flexion & lliopsoas and rectus femoris control and soft landing \\
Knee valgus with tibial femoris loading & Address dynamic control; decrease dynamic valgus & Lateral hip control on landing \\
Balance deficits & Proprioception drills & Dynamic balance training \\
Skill deficiency & Improve agility & Agility drills to address deceleration techniques and \\
& & core stability \\
\hline
\end{tabular}

Silvers and Mandelbaum ${ }^{25}$ developed the Santa Monica PEP ACL prevention program. This programme was used with two age cohorts: 14-18-year-old and 18-22-year-old female soccer players. It consisted of a 20 min warm-up to precede the normal training session. In the 2000 soccer season, a total of two ACL tears confirmed by MRI were reported for the intervention group for an incidence of 0.05 ACL injuries/athlete/1000 exposures. Thirty two ACL tears were reported for the control group (an incidence of 0.47 ACL injuries/athlete/1000 exposures). These results indicate an $88 \%$ overall reduction in ACL injury per individual athlete compared with a control athlete matched for skill and age. In year 2 (2001) of the study, four ACL tears were reported in the intervention group, with an incidence of 0.13 injuries/athlete/1000 exposures. Thirty five ACL tears were reported in the control group, with an incidence of 0.51 injuries/athlete/1000 exposures. This corresponds to an overall reduction of $74 \%$ in ACL tears in the intervention group compared with a control group matched for age and skill in year 2 .

The above study was followed by a randomised controlled trial using the PEP program in division I NCAA women's soccer teams in the 2002 fall season (Gilchrist J, et al, personal communication, 2004). Sixty one teams with 1429 athletes completed the study: 854 athletes participated in 35 control teams and 575 athletes participated in 26 intervention teams. No significant differences were noted between intervention and control athletes with regard to age, height, weight or history of ACL injury. After use of the PEP injury prevention program for one season, there were seven ACL injuries in the intervention athletes (rate of 0.14) and 18 in control athletes (rate of 0.25) $(p=0.15)$. There were no ACL injuries reported in the intervention athletes during practices compared with six in the control athletes $(0.10)(p=0.01)$. During game situations, the difference was not significant ( 7 vs 12 , respectively; $\mathrm{p}=0.76)$. Non-contact ACL injuries occurred at over three times the rate in control athletes $(n=10 ; 0.14)$ compared with intervention athletes $(\mathrm{n}=2 ; 0.04)(\mathrm{p}=0.06)$. Control athletes with a previous history of ACL injury had a recurrence 5 times more often than those in the intervention group ( 0.10 vs 0.02 ; $\mathrm{p}=0.06)$; this difference reached significance when limited to non-contact ACL injuries during the season (0.06 vs 0.00; $\mathrm{p}<0.05)$. There was a significant difference in the rate of ACL injuries in the second half of the season (weeks 6-11; intervention 0.00 vs control $0.18 ; \mathrm{p}<0.05$ ). This supports the concept that it takes 6-8 weeks for a biomechanical intervention programme to have a neuromuscular effect.

The similarities between the Santa Monica ACL prevention program and the intervention studies described above are numerous. Table 1 gives a summary of the programmes described above.

There is an emphasis on proper landing technique. Landing softly on the forefoot and rolling back to the rearfoot, engaging knee and hip flexion on landing and with lateral (cutting) manoeuvres, avoiding excessive genu valgum at the knee on landing and squatting, increasing hamstring, gluteus medius and hip abductor strength and addressing proper deceleration techniques are activities that seem to be inherent in each of the ACL prevention protocols. Table 2 summarises the relation of these components to specific risk factors for ACL injury.

The research discussed in this article should compel the medical community to pose the following questions:

- What is the true mechanism of ACL injury and is it sportspecific?

- Is the mechanism of ACL injury gender-specific or does it follow a similar biomechanical pattern?

- Does fatigue negate the preventive benefit of performing an ACL injury prevention programme?

- How do the successful ACL injury prevention programmes work and what are the common biomechanical threads between them?

These studies show the critical need for further randomised clinical trials on the relevance of fatigue, the age of implementation and timing of neuromuscular injury prevention programmes, and the role of fatigue with regard to injury.

\section{Authors' affiliations \\ Holly Jacinda Silvers, Santa Monica Orthopaedic Sports Medicine/ Research Foundation, Santa Monica, CA, USA \\ Bert R Mandelbaum, Santa Monica Orthopaedic and Sports Medicine Group, Santa Monica, USA}

Competing interests: None.

\section{REFERENCES}

1 Arendt E, Dick R. Knee injury patterns among men and women in collegiate basketball and soccer. Am J Sports Med 1995;23:694-701.

2 Seitz HMS, Wielke T, Vecsei V. Meniscus lesions after isolated anterior cruciate ligament rupture. Wien Klin Wochenschr 1996; 108:727-30.

3 Lohmander LSOA, Englund M, Roos $\mathrm{H}$. High prevalence of knee osteoarthritis, pain, and functional limitations in female soccer players twelve years after anterior cruciate ligament injury. Arthritis Rheum 2004;50:3145-52.

4 Shelbourne KDDT, Llootwyk T. The relationship between notch width and the risk for anterior cruciate ligament rupture. Am J Sports Med 1986;26:402-8.

5 Deppen RJLM. Efficacy of prophylactic knee bracing in high school football players. J Orthop Sports Phys Ther 1994;20:243-6.

6 Torg JS, Quedenfeld TC, Landau S. The shoe-surface interface and its relationship to football knee injuries. J Sports Med 1974;2:261-9.

7 Heidt RSDS, Cawley PW, et al. Differences in friction and torsional resistance in athletic shoe-turf surface interfaces. Am J Sports Med 1996;24:834-42.

8 Ekstrand J, Nigg BM. Surface related injuries in soccer. Sports Med 1989;8:56-62.

9 Huston LJVB, Ashton-Miller JA, Wojtys EM. Gender differences in knee angle when landing from a drop-jump. Am J Knee Surg 2001;14:215-19.

10 Scranton PE, Whitesel JP, Powell JW, et al. A review of selected noncontact anterior cruciate ligament injuries in the National Football League. Foot Ankle Int 1997; 18:772-6.

11 Orchard JW, Powell JW. Risk of knee and ankle sprains under various weather conditions in American football. Med Sci Sports Exerc 2003;35:1118-23.

12 Yu WDLS, Hatch JD, et al. Effect of estrogen on cellular metabolism of the human anterior cruciate ligament. Clin Orthop 1999;336:229-38.

13 Slauterbeck J, Clevenger C, Lundberg W, et al. Estrogen level alters the failure load of the rabbit anterior cruciate ligamant. J Orthop Res 1999;17:405-8. 
14 Woitys EMHL, Lindenfeld TN, Hewett TE, et al. Association between the menstrual cycle and anterior cruciate ligament injuries in female athletes. Am J Sports Med 1998;26:614-19.

15 Myklebust GMS, Holm I, Bahr R. A prospective cohort study of anterior cruciate ligament injuries in elite Norwegian team handball. Scand J Med Sci Sports 1998;8:149-53.

16 More RCKBT, Neiman R, et al. Hamstrings: an anterior cruciate ligament protagonist: an in vitro study. Am J Sports Med 1993;21:231-7.

17 McLean SG, Neal RJ, Myers PT, et al. Knee joint kinematics during the sidestep cutting maneuver: potential for injury in women. Med Sci Sports Exerc 1999;31:959-68.

18 McLean SGHX, Su A, Van Den Bogert AJ. Sagittal plane biomechanics cannot injure the ACL during sidestep cutting. Clin Biomech (Bristol, Avon) 2004;19:828-38.

19 Malinzak RACS, Kirkendall DT, Yu B, et al. A comparison of knee joint motion patterns between men and women in selected athletic tasks. Clin Biomech (Bristol, Avon) $2001 ; 16: 438-45$.

20 Henning CEGN. Injury prevention of the anterior cruciate ligament [videotape]. Mid-America Center for Sports Medicine, Wichita, Kansas, USA, 1990.

21 Caraffa ACG, Projetti M, Aisa G, et al. Prevention of anterior cruciate ligament injuries in soccer. A prospective controlled study of proprioceptive training. Knee Surg Sports Traumatol Arthrosc 1996;4:19-21.

22 Heweft TE, Liddenfeld TN, Riccobene JV, et al. The effect of neuromuscular training on the incidence of knee injury in female athletes. Am J Sports Med 1999;27:699-705.
23 Ettlinger DFJR, Shealy JE. A method to help reduce the risk of serious knee sprains incurred in alpine skiing. Am J Sports Med 1995;23:531-7.

24 Heidt RSSL, Carlonas RL, et al. Avoidance of soccer injuries with preseason conditioning. Am J Sports Med 2000;28:659-62.

25 Silvers HJ, Mandelbaum BR. Preseason conditioning to prevent soccer injuries in young women. Clin J Sport Med 2001;11:206.

26 Mandelbaum BR, Silvers HJ, et al. Prevention of ACL injury in the female soccer athlete: two-year follow-up. Am J Sports Med 2005;33:1003-10.

27 Myklebust G, et al. Prevention of anterior cruciate ligament injuries in female team handball players: a prospective intervention study over three seasons. Clin J Sport Med 2003;13:71-8.

28 Pfeiffer RP, Shea KG, Roberts D, et al. Lack of effect of a knee ligament injury prevention program on the incidence of noncontact anterior cruciate ligament injury. J Bone Joint Surg Am 2006;88:1769-74.

29 Söderman, et al. Balance board training: prevention of traumatic injuries of the lower extremities in female soccer players? A prospective randomised intervention study. Knee Surg Sports Traumatol Arthrosc 2000;8:321.

30 Olsen, Myklebust, et al. Prevention of knee and ankle injuries in youth team handball: a randomised controlled trial. BMJ 2005;330:449.

31 Wedderkopp, et al. Comparison of two intervention programmes in young female players in European handball with and without ankle disc. Scand J Med Sci Sports 2003;13:371-5. 